\title{
ON CONTACT SUB-PSEUDO-RIEMANNIAN ISOMETRIES
}

\author{
MAREK GROCHOWSKI AND WOJCIECH KRYŃSKI
}

\begin{abstract}
We study isometries in the contact sub-pseudo-Riemannian geometry. In particular we give an upper bound on the dimension of the isometry group of a general sub-pseudo-Riemannian manifold and prove that the maximal dimension is attained for the left invariant structures on the Heisenberg group.
\end{abstract}

\section{INTRODUCTION}

1.1. Results. Let $M$ be a smooth connected manifold. A sub-pseudoRiemannian structure on $M$ is a couple $(H, g)$ made up of a smooth bracket generating distribution $H$ of constant rank and a smooth pseudoRiemannian metric $g$ on $H$. At each point $q \in M, g$ can be represented as a diagonal matrix

$$
\operatorname{diag}(-1, \ldots,-1,+1, \ldots,+1)
$$

with, say, $l$ minuses. Clearly, by continuity, the number $l$ does not depend on a point $q$. It will be denoted $\operatorname{ind}(g)$ and called the index of the metric $(H, g)$.

A triple $(M, H, g)$ is called a sub-pseudo-Riemannian manifold. In particular, if $\operatorname{ind}(g)=0$ then $(M, H, g)$ is called a sub-Riemannian manifold. This case is best known and there are a lot of papers and books devoted to the sub-Riemannian geometry (see [1, 2, 3, 4, 14] and references therein). If $\operatorname{ind}(g)=1$ then $(M, H, g)$ is called a $s u b$ Lorentzian manifold (see [6, 8, 9, 11]). The sub-pseudo-Riemannian structures can be interpret as control systems [1, 5]. In particular the sub-Lorentzian structures give rise to a class of control-affine systems (c.f. [5, 6]).

In the sub-pseudo-Riemannian geometry we can ask the same questions as in the classical pseudo-Riemannian geometry. One of the most fundamental problems considered in the pseudo-Riemannian geometry is connected to calculations of the isometry group of a given pseudoRiemannian manifold. We shall consider a generalisation of this problem to the sub-pseudo-Riemannian case.

Date: August 24, 2018. 
Definition 1.1. Fix a sub-pseudo-Riemannain manifold $(M, H, g)$. A diffeomorphism $f: M \rightarrow M$ is called an isometry if

(D1) $f$ preserves the distribution, i.e. $f_{*}(H)=H$;

(D2) $f_{*}: H_{q} \rightarrow H_{f(q)}$ is a linear isometry for every $q \in M$, i.e. $g\left(f_{*}(v), f_{*}(w)\right)=g(v, w)$ for all $v, w \in H_{q}$.

The set of all isometries is a group (in fact a Lie group as it will become clear soon) and will be denoted $\mathfrak{I}(M, H, g)$. The component of the identity of this group is $\mathfrak{I}_{0}(M, H, g)$. Clearly $\operatorname{dim} \mathfrak{I}(M, H, g)=$ $\operatorname{dim} \mathfrak{I}_{0}(M, H, g)$. We shall assume that $H$ is a contact distribution meaning that it is locally given by the kernel of a contact one-form $\alpha$ satisfying

$$
(d \alpha)^{\wedge n} \wedge \alpha \neq 0
$$

where $\operatorname{dim} M=2 n+1$. In this case $(M, H, g)$ will be referred to as a contact sub-pseudo-Riemannian manifold. Our main result is the following

Theorem 1.2. Let $(M, H, g)$ be a contact sub-pseudo-Riemannian manifold. If $\operatorname{ind}(g)$ is even or $\operatorname{ind}(g)=\frac{1}{2} \mathrm{rk} H$ then

$$
\operatorname{dim} \mathfrak{I}(M, H, g) \leq \operatorname{dim} M+\left(\frac{1}{2} \mathrm{rk} H\right)^{2} .
$$

If $\operatorname{ind}(g)$ is odd and $\operatorname{ind}(g) \neq \frac{1}{2} \mathrm{rk} H$ then

$$
\operatorname{dim} \mathfrak{I}(M, H, g) \leq \operatorname{dim} M+\left(\frac{1}{2} \mathrm{rk} H-1\right)^{2}+1 .
$$

In Section 3, Proposition 3.6, we will show that the maximal dimension in (2) and (3) is attained by a left-invariant structure on the Heisenberg group. More precisely we will show that for any value of $\operatorname{ind}(g) \in\{0,1, \ldots, \mathrm{rk} H\}$ and any $t \leq \min \{\operatorname{ind}(g), \operatorname{rk} H-\operatorname{ind}(g)\}$ such that $\operatorname{ind}(g)-t$ is even there is a left-invariant structure such that

$$
\operatorname{dim} \mathfrak{I}(M, H, g)=\operatorname{dim} M+\left(\frac{1}{2} \mathrm{rk} H-t\right)^{2}+t^{2}
$$

In particular the maximal dimension in Theorem 1.2 is attained for $t=0$ if $\operatorname{ind}(g)$ is even, for $t=\operatorname{ind}(g)$ if $\operatorname{ind}(g)=\frac{1}{2} \operatorname{rk} H$ and for $t=1$ if $\operatorname{ind}(g)$ is odd and not equal $\frac{1}{2} \mathrm{rk} H$.

Let us point out here that invariants for the contact sub-pseudoRiemannian structures has been recently constructed in [7] (see also [1] for the sub-Riemannian case). The invariants vanish for the leftinvariant structures satisfying (4). 
1.2. Connections with control systems. Suppose that $(\Sigma) \dot{q}=$ $f(q, u)$ is a control system on a manifold $M$. By a symmetry of $(\Sigma)$ we mean a diffeomorphism of $M$ which maps the trajectories of $(\Sigma)$ onto trajectories of $(\Sigma)$. It turns out that the described results concerning isometry groups of sub-pseudo-Riemannian manifolds can be formulated in terms of symmetries of certain control systems.

Indeed, suppose that $(M, H, g)$ is a sub-pseudo-Riemannian manifold with $\operatorname{ind}(g)=l$ and $\mathrm{rk} H=k$. By a timelike (resp. spacelike) curve on $(M, H, g)$ we mean every absolutely continuous curve $\gamma:[a, b] \rightarrow M$ such that $\dot{\gamma} \in H_{\gamma(t)}$ and moreover $g(\dot{\gamma}(t), \dot{\gamma}(t))<0$ (resp. $g(\dot{\gamma}(t), \dot{\gamma}(t))>0)$ for almost every $t \in[a, b]$. Suppose that $X_{1}, \ldots, X_{k}$ is an orthonormal basis for $(H, g)$ defined on an open set $U$ such that $g\left(X_{i}, X_{i}\right)=-1$ for $i=1, \ldots, l$ and $g\left(X_{i}, X_{i}\right)=1$ for $i=l+1, \ldots, k$. Timelike (resp. spacelike) curves in $U$ with unit speed parametrization can be represented as solutions to the following control system

$$
\dot{q}=\sum_{i=1}^{k} u_{i} X_{i}(q)
$$

with the set of control parameters equal to

$$
\mathcal{U}=\left\{\left(u_{1}, \ldots, u_{k}\right) \mid-\sum_{i=1}^{l} u_{i}^{2}+\sum_{i=l+1}^{k} u_{i}^{2}=-1\right\},
$$

or

$$
\mathcal{U}=\left\{\left(u_{1}, \ldots, u_{k}\right) \mid-\sum_{i=1}^{l} u_{i}^{2}+\sum_{i=l+1}^{k} u_{i}^{2}=1\right\},
$$

respectively, where controls are supposed to be measurable and bounded. Now, it is easy to show that in both cases the symmetries of (15) coincide with the isometry group $\mathfrak{I}\left(U, H_{\mid U}, g\right)$. One can also consider the sets of null, or nonspecelike curves defined by similar control systems. However in the latter cases the isometry group $\mathfrak{I}\left(U, H_{\mid U}, g\right)$ is only a subgroup of all symmetries.

In the sub-Lorentzian setting the future directed nonspacelike curves can be described by a control-affine system. To be precise, by a time orientation of a sub-Lorentzian manifold $(M, H, g)$ we understand a timelike vector field $X$ on $M$ (i.e. $X(q) \in H_{q}$ and $g(X(q), X(q))<0$ for every $q \in M)$. A nonspacelike curve $\gamma:[a, b] \rightarrow M$ is said to be future directed if $g(\dot{\gamma}(t), X(\gamma(t)))<0$ a.e. on $[a, b]$ (c.f. [5, 6]). Suppose that $X$ is a fixed time orientation and $U$ is an open set on which there exist spacelike vector fields $X_{2}, \ldots, X_{k}$ such that $X, X_{2}, \ldots, X_{k}$ form an orthonormal basis for $(H, g)$ over $U$. As it is explained in [5] every 
nonspacelike future directed curve in $U$ is, up to a reparameterization, a trajectory of the control-affine system

$$
\dot{q}=X+\sum_{i=2}^{k} u_{i} X_{i}(q),
$$

where the set of control parameters equals the unit ball in $\mathbb{R}^{k-1}$ centered at zero. Now it is clear that $\mathfrak{I}\left(U, H_{\mid}, g\right)$ is a group of symmetries of the system (6). We refer to [1, 5, 6] for more information on the mentioned control systems, the corresponding reachable sets and optimal solutions to the control problems.

1.3. The content of the paper. The paper is organised as follows. In Section 2 we formulate and explain basic facts and assumptions that we use later on. We show that $g$ can be extended to a metric on $T M$ in a canonical way and exploit this fact to prove that $\mathfrak{I}(M, H, g)$ is a Lie group (Theorem 2.2). Moreover, we introduce a canonical symplectic structure on $H$.

Sections 3 and 4 are devoted to special classes of sub-pseudo-Riemannian metrics. In Section 3 we consider sub-pseudo-Riemannian structures satisfying an additional compatibility condition. In the Riemannian signature the condition guarantees that $H$ caries an almost complex structure. In Section 4 we consider so-called regular structures, which include all sub-Riemannian and sub-Lorentzian metrics in neighbourhoods of generic points. We estimate from above dimensions of the isometry groups for these spacial classes of structures (Theorems 3.3 and 4.2). Moreover, we construct examples with isometry groups of dimension given by formula (4).

Section 5 contains the proof of Theorem 1.2. The main idea relies on the calculation of the Tanaka prolongations of certain graded Lie algebras and on the results of Kruglikov [12, 13] that extend Tanaka's theory to the case of non-constant symbol algebras.

\section{Contact sub-Pseudo-Riemannian structures}

2.1. Extended metric. Let $(M, H, g)$ be a contact sub-pseudo-Riemannian manifold of dimension $2 n+1$. Fix $q \in M$ and assume that $H=\operatorname{ker} \alpha$ in a neighbourhood of $q$, where $\alpha$ satisfies (1). The contact form $\alpha$ defines the Reeb vector field $X_{\alpha}$ by the conditions

$$
X_{\alpha} \in \operatorname{ker} d \alpha, \quad \alpha\left(X_{\alpha}\right)=1 .
$$

It follows that $X_{\alpha}$ is transverse to $H$. Clearly $X_{\alpha}$ depends essentially on the choice of $\alpha$ and the one-form is not unique. However it can be normalised in the following way. Let $\left(X_{1}, \ldots, X_{2 n}\right)$ be an orthonormal 
frame of $H$ in a neighbourhood of $q$. Then, multiplying $\alpha$ by a smooth function, we can impose the condition

$$
\left|(d \alpha)^{\wedge n}\left(X_{1}, \ldots, X_{2 n}\right)\right|=1,
$$

which does not depend on the choice of an orthonormal frame. As a result, we get a canonical contact form $\alpha$ given up to a multiplication by \pm 1 in the neighbourhood of $q \in M$. We shall see later that for oriented structures one can rid off this ambiguity and get a unique canonical global contact form $\alpha$ on $M$. However, we do not need the uniqueness at this point and using the two normalised contact forms in a neighbourhood of any point $q \in M$ we are able to extend $g$ from $H$ to a metric $G$ on $T M$. Indeed, we set

$$
\left.G\right|_{H \times H}=g
$$

and

$$
G\left(X_{\alpha}, X_{\alpha}\right)=1, \quad G\left(X_{\alpha}, H\right)=0,
$$

where $\alpha$ is a contact form satisfying (8) and $X_{\alpha}$ is the Reeb vector field corresponding to $\alpha$. Since $\alpha$ is given up to a sign, we conclude that $X_{\alpha}$ is given up to a sign too. However, $G$ does not depend on the sign and we obtain unique $G$ in a neighbourhood of each point $q \in M$. The uniqueness implies that $G$ must coincide on overlaps of neighbourhoods of different points. Thus, we get a globally defined metric $G$ on $M$ which is canonically determined by the structure $(H, g)$. Since any isometry preserves the form $\alpha$ up to a sign, we have proved the following

Proposition 2.1. If $f: M \rightarrow M$ is an isometry of a sub-pseudoRiemannian structure $(H, g)$ then $f^{*} G=G$. Thus $f$ is an isometry of $G$, too.

We shall denote by $\mathfrak{I}(M, G)$ the group of isometries of $(M, G)$. We refer to [7] for more detailed discussion on the possible extensions of $g$. Let $O_{G}(M)$ be the orthonormal frame bundle for $G$. We define $O_{H, g}(M)$, the orthonormal frame bundle of $(H, g)$, as a sub-bundle of $O_{G}(M)$ consisting of points $\left(q ; v_{1}, \ldots, v_{2 n}, v_{0}\right)$ such that $\left(v_{1}, \ldots, v_{2 n}\right)$ is an orthonormal basis of $H_{q}$. In particular, it follows that $v_{0}=X_{\alpha}(q)$ where $\alpha$ is one of the two contact forms normalised by (8) in a neighbourhood of $q$. Now, any pseudo-Riemannian isometry $f \in \mathfrak{I}(M, G)$ is uniquely determined by the values of $f(q)$ and $f_{*}(q)$ where $q$ is an arbitrary fixed point in $M[10$. Since $\mathfrak{I}(M, H, g)$ is a closed subgroup of $\mathfrak{I}(M, G)$ we get 
Theorem 2.2. $\mathfrak{I}(M, H, g)$ is a Lie group with respect to the opencompact topology. Moreover any contact sub-pseudo-Riemannian isometry $f \in \mathfrak{I}(M, H, g)$ is uniquely determined by two values: $f(q)$ and $f_{*}(q)$, where $q \in M$ is an arbitrarily fixed point. Additionally, fixing an arbitrary point $\left(q ; v_{1}, \ldots, v_{2 n}, v_{0}\right) \in O_{H, g}(M)$, the mapping

$$
f \longmapsto\left(f(q) ; f_{*}\left(v_{1}\right), \ldots, f_{*}\left(v_{2 n}\right), f_{*}\left(v_{0}\right)\right)
$$

defines an embedding of $\mathfrak{I}(M, H, g)$ to $O_{H, g}(M)$.

Proof. Follows from the fact that $\mathfrak{I}(M, G)$ is a Lie group [10] and its subgroup $\mathfrak{I}(M, H, g)$ is closed in $\mathfrak{I}(M, G)$.

2.2. Orientation. Let $(M, H, g)$ be a contact sub-pseudo-Riemannian manifold of dimension $2 n+1$. We shall say that the structure is oriented if the two vector bundles $T M$ and $H$ are oriented (see [6] for various notions of orientations related to the casual decomposition of the distribution under consideration). We shall see that the structure is oriented if and only if there is a global contact form annihilating $H$. There are two cases depending on the parity of $n$.

If $n$ is even then $(d \alpha)^{\wedge n}$ is independent of the sign of $\alpha$. Conversely, the sign of $d \alpha^{\wedge n} \wedge \alpha$ changes if the sign of $\alpha$ changes. Thus, on the one hand, $H$ is canonically oriented, because fixing an open cover $\left\{U_{s}\right\}_{s \in \Sigma}$ of $M$ and local contact forms $\left\{\alpha_{s}\right\}_{s \in \Sigma}$ annihilating $H$ on $U_{s}$ we can rescale the forms such that $\left(d \alpha_{s}\right)^{\wedge n}$ glue to a global $2 n$-form non-degenerate on $H$. On the other hand, $M$ is oriented if and only if there is a global contact form annihilating $H$. Indeed, if $\alpha$ is a global contact form then $d \alpha^{\wedge n} \wedge \alpha$ defines an orientation of $M$. Conversely, if an orientation of $M$ is given then we can rescale local contact forms $\left\{\alpha_{s}\right\}_{s \in \Sigma}$ annihilating $H$ such that $d \alpha_{s}^{\wedge n} \wedge \alpha_{s}$ agree with the orientation. Clearly, such rescaled one-forms must coincide on the intersections of domains $U_{s}$. Thus, they define a global one-form on $M$.

If $n$ is odd then $(d \alpha)^{\wedge n} \wedge \alpha$ is independent of the sign of $\alpha$. Conversely, the sign of $d \alpha^{\wedge n}$ changes if the sign of $\alpha$ changes. Thus, similarly to the case of even $n$, we deduce that on the one hand $M$ is canonically oriented, and, on the other hand, $H$ is oriented if and only if there is global contact form annihilating $H$.

Suppose that $(M, H, g)$ is oriented. In view of the discussion above we can assume that the orientation of $M$ is given by $d \alpha^{\wedge n} \wedge \alpha$ and the orientation of $H$ is given by $d \alpha^{\wedge n}$, where $\alpha$ is a global contact form. Then $\alpha$ is given up to a multiplication by a positive function. However, we can choose the unique one which satisfies the normalisation condition (8). We shall call this form the canonical contact form of an 
oriented contact structure. The canonical contact form satisfies

$$
(d \alpha)^{\wedge n}\left(X_{1}, \ldots, X_{2 n}\right)=1 .
$$

where $\left(X_{1}, \ldots, X_{2 n}\right)$ is an arbitrary positively oriented orthonormal frame of $H$.

If $(M, H, g)$ is oriented then we shall consider isometries preserving the orientation.

2.3. Symplectic structure. Assume that $(M, H, g)$ is an oriented sub-pseudo-Riemannian manifold and let $\alpha$ be the canonical contact form. We introduce

$$
\omega=-\left.d \alpha\right|_{H}
$$

Then $\omega$ is a symplectic structure on $H$ canonically defined by $\alpha$.

Proposition 2.3. If $f: M \rightarrow M$ is an isometry of an oriented subpseudo-Riemannian structure then $f^{*} \omega=\omega$.

The pair $(g, \omega)$ defines the operator $J: H \rightarrow H$ by the formula

$$
\omega_{q}(v, w)=g\left(J_{q}(v), w\right), \quad q \in M, \quad v, w \in H_{q} .
$$

The eigenvalues of $J$ are basic invariants of the structure $(H, g)$ at each point $q \in M$. We shall analyse the structure of eigenspaces of $J$ using the Kronecker theorem that gives normal forms of pencils of matrices. Precisely, we apply the Kronecker theorem to the pair $(g, \omega)$, i.e. to a pair of a symmetric and a skew-symmetric bi-linear forms. For a detailed analysis of this particular case of the Kronecker theorem we refer to [16]. We shall use later the following properties of eigenvalues of $J$ :

(P1) if $\lambda$ is an eigenvalue of $J$ then also $-\lambda$ is;

(P2) if $\lambda$ has non-zero real part then $g$ restricted to the corresponding eigenspace $H_{\lambda}$ is degenerate: $\left.g\right|_{H_{\lambda} \times H_{\lambda}}=0$;

(P3) if $\lambda$ is purely imaginary and $\left.g\right|_{H_{\lambda} \times H_{\lambda}} \neq 0$ then $\operatorname{dim} H_{\lambda}=2$ and $g$ on $H_{\lambda}$ is definite; in this case $b=|\lambda|$ is called a frequency (c.f. [1]).

Thus, at each $q \in M$ the distribution $H$ decomposes as follows

$$
H=\hat{H} \oplus \tilde{H}
$$

where

$$
\hat{H}=H_{\lambda_{1}} \oplus \cdots \oplus H_{\lambda_{s}} .
$$

and all $H_{\lambda_{i}}, i=1, \ldots, s$ are two dimensional and correspond to purely imaginary eigenvalues (some may repeat). Additionally $\tilde{H}$ is of dimension $2 n-2 s$ and ind $\left.g\right|_{\tilde{H} \times \tilde{H}}=n-s$. Moreover $\tilde{H}$ decomposes further 
to eigenspaces which are null with respect to $g$ and appear in pairs $H_{\lambda} \oplus H_{-\lambda}$.

According to (12) and (13) $J$ has the following form

$$
J=\left(\begin{array}{cccccc}
0 & -b_{1} & \cdots & 0 & 0 & 0 \\
b_{1} & 0 & \cdots & 0 & 0 & 0 \\
\vdots & \vdots & \ddots & \vdots & \vdots & \vdots \\
0 & 0 & \cdots & 0 & -b_{s} & 0 \\
0 & 0 & \cdots & b_{s} & 0 & 0 \\
0 & 0 & \cdots & 0 & 0 & \tilde{J}
\end{array}\right)
$$

where $\left(b_{1}, \ldots, b_{s}\right)$ are frequencies and $\tilde{J}$ is a matrix of dimension $(2 n-$ $2 s) \times(2 n-2 s)$.

Note that if the signature of $g$ is Riemannian then there is no term $\tilde{H}$ in the decomposition and we have $n$ frequencies that satisfy (c.f. [1])

$$
\prod_{i=1}^{n} b_{i}=1
$$

due to (10). On the other hand if index of $g$ is odd then $\tilde{H}$ always appears. In a very particular case it may occur that all eigenvalues of $\tilde{J}$ are real and the corresponding eigenspaces are one dimensional. Then

$$
\tilde{J}=\left(\begin{array}{ccccc}
0 & c_{1} & \cdots & 0 & 0 \\
c_{1} & 0 & \cdots & 0 & 0 \\
\vdots & \vdots & \ddots & \vdots & \vdots \\
0 & 0 & \cdots & 0 & c_{t} \\
0 & 0 & \cdots & c_{t} & 0
\end{array}\right)
$$

for some $\left(c_{1}, \ldots, c_{t}\right)$ where $t=n-s$ and

$$
\left(\prod_{i=1}^{s} b_{i}\right)\left(\prod_{i=1}^{t} c_{i}\right)=1,
$$

due to (10) again. In particular if $(H, g)$ is a sub-Lorentzian structure in dimension 3 then the three properties (P1)-(P3) of $J$ and the normalisation condition (10) imply that $J=\tilde{J}$ and the two null directions in $H$ are eigenspaces with real eigenvalues \pm 1 . Therefore, we can choose an orthonormal frame such that $J=\tilde{J}$ and

$$
\tilde{J}=\left(\begin{array}{ll}
0 & 1 \\
1 & 0
\end{array}\right)
$$


2.4. Reduction. Let $(H, g)$ be an oriented sub-pseudo-Riemannian contact structure on $M$. Then the symplectic structure $\omega$ reduces $O_{H, g}(M)$ to the sub-bundle $O_{H, g, \omega}(M)$ of frames which put $(g, \omega)$ into the canonical Kronecker form. Thus, $O_{H, g, \omega}(M)$ consists of points $\left(q ; v_{1}, \ldots, v_{2 m}, v_{0}\right) \in O_{H, g}$ such that $\left(v_{1}, \ldots, v_{2 m}\right)$ is a positively oriented, orthonormal basis of $H_{q}$ and $J_{q}$ in this basis is given by (14). Moreover, we assume that $v_{0}=X_{\alpha}(q)$, where $X_{\alpha}$ is the Reeb vector field corresponding to the canonical contact form. Then the following group acts freely and transitively on $O_{H, g}(M)_{q}$

$$
\mathcal{G}_{g, \omega}(q)=\left\{\left(\begin{array}{ll}
A & 0 \\
0 & 1
\end{array}\right) \mid A \in O\left(g_{q}\right) \cap S p\left(\omega_{q}\right)\right\},
$$

where $O\left(g_{q}\right)$ is the subgroup of $G L\left(H_{q}\right)$ preserving $g_{q}$ and $S p\left(\omega_{q}\right)$ is the subgroup of $G L\left(H_{q}\right)$ preserving $\omega_{q}$. Of course $O\left(g_{q}\right) \simeq O(l, 2 n-l)$, where $l=\operatorname{ind}(g)$ and $O(l, 2 n-l)$ is the standard group of matrices preserving a metric of index $l$ and $S p\left(\omega_{q}\right) \simeq S p(2 n)$, where $S p(2 n)$ is the group of matrices preserving the standard symplectic form given by

$$
\Omega=\left(\begin{array}{cc}
0 & -I_{n} \\
I_{n} & 0
\end{array}\right)
$$

where $I_{n}$ is the $n \times n$ identity matrix. Note that automatically $O\left(g_{q}\right) \cap$ $S p\left(\omega_{q}\right) \subset S O\left(g_{q}\right)$, because the orientation is defined in terms of $\omega_{q}$.

The intersection $O\left(g_{q}\right) \cap S p\left(\omega_{q}\right)$ essentially depends on $g$ and $\omega$ at a given point and the groups $\mathcal{G}_{g, \omega}(q)$ may be not isomorphic for different $q$. Actually, we shall show later that the dimension of $\mathcal{G}_{g, \omega}(q)$ depends on the decomposition of $J_{q}$ into the sum of eigenspaces.

\section{Compatibility CONDition}

3.1. Isometries of compatible structures. We will consider a particular class of oriented contact sub-pseudo-Riemannian structures such that $g$ and $\omega$ are compatible. One expects that the most symmetric structures are among this class.

Definition 3.1. Let $(M, H, g)$ be an oriented sub-pseudo-Riemannian manifold and let $\omega$ be the corresponding symplectic structure on $H$. Then $g$ and $\omega$ are compatible if in a neighbourhood of any $q \in M$ there is a frame which is mutually orthonormal with respect to $g$ and symplectic with respect to $\omega$. The sub-pseudo-Riemannian structure satisfies the compatibility condition if $g$ and $\omega$ are compatible.

Note that in the case of compatible structures with $g$ being Riemannian, $J$ is an almost complex structure on $H$. Similarly, in the 
case of compatible structures with $\operatorname{ind}(g)=\frac{1}{2} \mathrm{rk} H, J$ is a para-CR structure, provided that there are no purely imaginary eigenvalues of $J$. In general, the compatibility condition can be expressed in terms of frequencies.

Proposition 3.2. An oriented sub-pseudo-Riemannian structure satisfies the compatibility condition if and only if the frequencies in (14) satisfy $b_{i}=1, i=1, \ldots, s$ and $\tilde{J}$ is of the form (15) with $c_{i}=1$, $i=1, \ldots, t$.

Proof. Follows directly from the definition.

The bundle $O_{H, g, \omega}(M)$ for a structure $(H, g)$ satisfying the compatibility condition is the bundle of frames that are mutually orthonormal with respect to $g$ and symplectic with respect to $\omega$. Proposition 3.2 implies that under the compatibility condition all $\mathcal{G}_{g, \omega}(q), q \in M$, are isomorphic, because $J_{q}$ depends smoothly on $q$ and $M$ is connected. Thus $O_{H, g, \omega}(M)$ is a principal bundle with the structure group isomorphic to $\mathcal{G}_{g, \omega}(q)$ for any fixed $q \in M$. The structure group will be simply denoted $\mathcal{G}_{g, \omega}$. Moreover, Proposition 2.3 implies that the embedding (9) restricted to the component of identity $\mathfrak{I}_{0}(M, H, g)$ takes values in $O_{H, g, \omega}(M)$. Precisely, fixing $\left(q ; v_{1}, \ldots, v_{2 n}, v_{0}\right) \in O_{H, g, \omega}$ we get that

$$
f \longmapsto\left(f(q) ; f_{*}\left(v_{1}\right), \ldots, f_{*}\left(v_{2 n}\right), f_{*}\left(v_{0}\right)\right)
$$

defines an embedding of $\mathfrak{I}_{0}(M, H, g)$ to $O_{H, g, \omega}(M)$. This embedding permits to prove

Theorem 3.3. Let $(M, H, g)$ be an oriented contact sub-pseudo-Riemannian manifold satisfying the compatibility condition. Then

$$
\operatorname{dim} \mathfrak{I}(M, H, g) \leq 2 n+1+s^{2}+(n-s)^{2},
$$

where $\operatorname{dim} M=2 n+1$ and $s=\frac{1}{2} \operatorname{rk} \hat{H}$ is the multiplicity of $i=\sqrt{-1}$ as an eigenvalue of the endomorphism $J$. Moreover, the parity of $n-s$ equals to the parity of $\operatorname{ind}(g)$.

Proof. We recall that $g$ restricted to any two-dimensional component $H_{\lambda}$ of $\hat{H}$ in the decomposition (13) is definite. Additionally $g$ restricted to $\tilde{H}$ has index equal to $\frac{1}{2}$ rk $\tilde{H}$. Thus

$$
\operatorname{ind}(g)=\frac{1}{2} \operatorname{rk} \tilde{H} \bmod 2
$$

and since $\frac{1}{2} \mathrm{rk} \tilde{H}=n-s$ the last statement of the Theorem follows.

Therefore, it is sufficient to compute the dimension of $\mathcal{G}_{g, \omega}$ in order to complete the proof, because the existence of the embedding (18) 
implies

$$
\operatorname{dim} \mathfrak{I}_{0}(M, H, g) \leq \operatorname{dim} M+\operatorname{dim} \mathcal{G}_{g, \omega},
$$

and $\operatorname{dim} \mathfrak{I}(M, H, g)=\operatorname{dim} \mathfrak{I}_{0}(M, H, g)$. The result follows from the following general Lemma that will be also used later in the proof of Theorem 1.2 .

Lemma 3.4. Let $s=\frac{1}{2} \operatorname{rk} \hat{H}$ and $t=\frac{1}{2} \operatorname{rk} \tilde{H}$, where $\hat{H}$ and $\tilde{H}$ are defined by the decomposition (12) of the operator $J$ for a pair $(g, \omega)$ of arbitrary non-degenerate symmetric and skew-symmetric bi-linear forms on $H, \operatorname{rk} H=2 n$. Then

$$
\operatorname{dim}(O(g) \cap S p(\omega))=s^{2}+t^{2} .
$$

Proof. We shall consider the Lie algebra $\mathfrak{g}$ of $O(g) \cap S p(\omega)$, because $\operatorname{dim} \mathfrak{g}=\operatorname{dim}(O(g) \cap S p(\omega))$. Let $A=\left(a_{i, j}\right)_{i, j=1, \ldots, 2 n} \in \mathfrak{g}$. Then, according to the decomposition (12) $A$ decomposes into the following block form

$$
A=\left(\begin{array}{cc}
B & D \\
D^{\prime} & C
\end{array}\right)
$$

where $A$ is of dimension $2 s \times 2 s, D$ is of dimension $2 s \times 2 t, D^{\prime}$ is of dimension $2 t \times 2 s$ and $C$ is of dimension $2 t \times 2 t$. Now, since $A$ preserves the eigenspces of $J$ and $g$ restricted to the eigenspaces, we get from properties (P2) and (P3) of $J$ that $D=D^{\prime}=0$. Thus we shall estimate the possible number of independent entries of $B$ and $C$.

Let us consider $B$ first. In order to get an estimate we can assume that all $b_{i}=1$. Otherwise $B$ would decompose into smaller blocks. So, we can choose a basis in $\hat{H}$ such that $g$ is diagonal and $\omega$ is a standard symplectic form. Then, on the one hand $B$ is completely determined by entries above the diagonal, because $B \in \mathfrak{s o}(\hat{l}, 2 s-\hat{l})$, where $\hat{l}=$ ind $\left.g\right|_{\hat{H} \times \hat{H}}$. On the other hand $B$ is completely determined by the entries above the anti-diagonal (including the anti-diagonal itself), because $B \in \mathfrak{s p}(2 s)$. Thus, $B$ has $s^{2}$ independent entries.

Now, let us consider $C$. We have ind $\left.g\right|_{\tilde{H} \times \tilde{H}}=\frac{1}{2} \operatorname{rk} \tilde{H}=t$. Thus, we can assume that $g$ is diagonal

$$
\left.g\right|_{\tilde{H} \times \tilde{H}}=\operatorname{diag}(\underbrace{-1, \ldots,-1}_{t}, \underbrace{+1, \ldots,+1}_{t}) .
$$

Moreover, due to (P2), we can assume $\left.\omega\right|_{\tilde{H} \times \tilde{H}}$ is given by a nondegenerate skew-symmetric matrix of the form

$$
\left.\omega\right|_{\tilde{H} \times \tilde{H}}=\left(\begin{array}{cc}
0 & \tilde{\omega}_{12} \\
-\tilde{\omega}_{12}^{T} & 0
\end{array}\right),
$$


where $\tilde{\omega}_{12}$ is of dimension $t \times t$ (c.f. the normal forms in [16]). Let

$$
C=\left(\begin{array}{ll}
C_{11} & C_{12} \\
C_{21} & C_{22}
\end{array}\right)
$$

where all $C_{i j}$ are of dimension $t \times t$. Then $C_{11}$ and $C_{22}$ are skewsymmetric and $C_{12}=C_{21}^{T}$ due to $C \in \mathfrak{s o}(t, t)$. Moreover $C_{11}=$ $\tilde{\omega}_{12} C_{22} \tilde{\omega}_{12}^{-1}$ and $C_{12}=\tilde{\omega}_{12} C_{12}^{T} \tilde{\omega}_{12}^{-1}$ due to $C \in \mathfrak{s p}(\omega)$. Thus, $C$ has at most $t^{2}$ independent entries.

Finally let us notice that the maximal dimensions are attained if all $b_{i}=1$ and $\tilde{J}$ is of the form (15) with all $c_{i}=1$.

3.2. Left invariant structures on the Heisenberg group. We will show that the upper bound on the dimension of the group of isometries from Theorem 3.3 is attained. In particular, taking into account the parity of ind $(g)$, we will show that there are structures with the isometry groups of dimensions as in Theorem 1.2 and formula (44).

To this end we consider left-invariant structures on the Heisenberg group. We recall that the Heisenberg group is realised as the space $\mathbb{R}^{2 n+1}$ with the contact distribution $H$ defined as follows. Suppose we have coordinates $x_{1}, \ldots, x_{n}, y_{1}, \ldots, y_{n}, z$ on $\mathbb{R}^{2 n+1}$ which will be denote by $(x, y, z)$ for short. Let

$$
X_{i}=\frac{\partial}{\partial x_{i}}+\frac{1}{2} y_{i} \frac{\partial}{\partial z}, \quad Y_{i}=\frac{\partial}{\partial y_{i}}-\frac{1}{2} x_{i} \frac{\partial}{\partial z}
$$

$i=1, \ldots, n$. Define $H$ to be

$$
H=\operatorname{span}\left\{X_{1}, Y_{1}, \ldots, X_{n}, Y_{n}\right\}
$$

We equip $\left(\mathbb{R}^{2 n+1}, H\right)$ with metric $g$ by declaring the frame $\left(X_{1}, Y_{1}\right.$, $\left.\ldots, X_{n}, Y_{n}\right)$ to be orthonormal and such that

$$
g\left(X_{i}, X_{i}\right)=t_{i}, \quad g\left(Y_{i}, Y_{i}\right)=s_{i}
$$

where $t_{i}, s_{i} \in\{-1,1\}$ depending on the signature of $g$. The vector fields (19) are left invariant fields with respect to the standard multiplication on the Heisenberg group

$$
\begin{aligned}
& \left(x_{1}, \ldots, x_{n}, y_{1}, \ldots, y_{n}, z\right) *\left(x_{1}^{\prime}, \ldots, x_{n}^{\prime}, y_{1}^{\prime}, \ldots, y_{n}^{\prime}, z^{\prime}\right)= \\
& \left(x_{1}+x_{1}^{\prime}, \ldots x_{n}+x_{n}^{\prime}, y_{1}+y_{1}^{\prime}, \ldots, y_{n}+y_{n}^{\prime}, z+z^{\prime}+\frac{1}{2} \sum_{i=11}^{n}\left(y_{i} x_{i}^{\prime}-y_{i}^{\prime} x_{i}\right)\right) .
\end{aligned}
$$


The symplectic structure on $H$ is the standard one

$$
\omega=\sum_{i=1}^{n} d x_{i} \wedge d y_{i}
$$

Take a matrix $\sigma \in S p(\omega) \cap O(g)$. We will show that the map $f_{\sigma}: \mathbb{R}^{2 n+1} \rightarrow \mathbb{R}^{2 n+1}$ defined by

$$
f_{\sigma}(x, y, z)=\left(\sigma \cdot(x, y)^{T}, z\right)
$$

is an isometry. Denote $f_{\sigma}=\left(f_{\sigma}^{1}, \ldots, f_{\sigma}^{2 n}, f_{\sigma}^{2 n+1}\right)$. Then

$$
f_{\sigma}^{i}(x, y, z)=\sum_{j=1}^{n}\left(\sigma_{i, j} x_{j}+\sigma_{i, n+j} y_{j}\right)
$$

for $i=1, \ldots, 2 n$. First we have

Lemma 3.5. For any $\sigma \in S p(\omega)$

$$
f_{\sigma *}\left(X_{i}\right)(x, y, z)=\sum_{j=1}^{n} \sigma_{j, i} X_{j}\left(f_{\sigma}(x, y, z)\right)+\sum_{j=1}^{n} \sigma_{n+j, i} Y_{j}\left(f_{\sigma}(x, y, z)\right)
$$

and

$$
f_{\sigma *}\left(Y_{i}\right)(x, y, z)=\sum_{j=1}^{n} \sigma_{j, n+i} X_{j}\left(f_{\sigma}(x, y, z)\right)+\sum_{j=1}^{n} \sigma_{n+j, n+i} Y_{j}\left(f_{\sigma}(x, y, z)\right) .
$$

In particular, $f_{\sigma}$ preserves $H$.

Proof. We will prove the first equality only. Using (22) we directly compute

$$
f_{\sigma_{*}}\left(X_{i}\right)=\sum_{j=1}^{n} \sigma_{j, i} \frac{\partial}{\partial x_{j}}+\sum_{j=1}^{n} \sigma_{n+j, i} \frac{\partial}{\partial y_{j}}+\frac{1}{2} y_{i} \frac{\partial}{\partial z} .
$$

Now, it is enough to show that

$$
\sum_{j=1}^{n} \sigma_{j, i} f_{\sigma}^{n+j}(x, y, z)-\sum_{j=1}^{n} \sigma_{n+j, i} f_{\sigma}^{j}(x, y, z)=y_{i} .
$$

However, using (22) again, we have

$$
\begin{aligned}
& \sum_{j=1}^{n} \sigma_{j, i} f_{\sigma}^{n+j}(x, y, z)-\sum_{j=1}^{n} \sigma_{n+j, i} f_{\sigma}^{j}(x, y, z)= \\
& \sum_{j, k=1}^{n}\left(\sigma_{n+j, k} \sigma_{j, i}-\sigma_{j, k} \sigma_{n+j, i}\right) x_{k}+\sum_{j, k=1}^{n}\left(\sigma_{n+j, n+k} \sigma_{j, i}-\sigma_{j, n+k} \sigma_{n+j, i}\right) y_{k}
\end{aligned}
$$

and the lemma follows from the fact that $\omega$ is the standard symplectic form, i.e. $\sigma \Omega \sigma^{T}=\Omega$, where $\Omega$ is given by (17). 
Now, we can prove the following

Proposition 3.6. The group of orientation preserving isometries of the left-invariant contact sub-pseudo-Riemannian structure defined above on the Heisenberg group is isomorphic to

$$
\mathbb{R}^{2 n+1} \ltimes(S p(\omega) \cap O(g)) .
$$

Proof. If $\sigma \in S p(\omega) \cap O(g)$ then the formulae for $f_{\sigma_{*}}\left(X_{i}\right)$ and $f_{\sigma_{*}}\left(Y_{i}\right)$ in Lemma 3.5 imply that $f_{\sigma}$ is an isometry. Thus any $\sigma \in S p(\omega) \cap O(g)$ defines an isometry of $(H, g)$ and we get the second factor in (23). The first factor in (23) comes from left translations. There can not be more isometries due to the embedding (18).

Remark 3.7. Let us remark that the full group of isometries is isomorphic to the product $\mathbb{R}^{2 n+1} \ltimes(\tilde{S p}(\omega) \cap O(g))$ where $\tilde{S p}(\omega)$ is the group preserving $\omega$ up to the sign.

\section{Regularity CONDITION}

4.1. Isometries of regular structures. Before proceeding to the general case announced in Theorem 1.2 we will describe a class of subpseudo-Riemannian structures which generalize those satisfying the compatibility condition but, at the same time, simple enough so that the isometry groups can be explicitly computed.

Definition 4.1. Let $(M, H, g)$ be a contact sub-pseudo-Riemannian manifold of dimension $2 n+1$. The metric $(H, g)$ is said to satisfy the regularity condition if there exists a global orthonormal frame $X_{1}, \ldots, X_{2 n}$ with respect to which the symplectic form $\omega$ on $H$ can be written as

$$
\omega=\sum_{i=1}^{n} b_{i} \alpha^{i} \wedge \alpha^{n+i}
$$

where $\alpha^{1}, \ldots, \alpha^{2 n}$ is the co-frame dual to $X_{1}, \ldots, X_{2 n}$, and $b_{1}, \ldots, b_{n}$ are smooth functions such that there exist positive integers $k_{1}, \ldots, k_{r}$, $k_{1}+\cdots+k_{r}=n$, for which

$$
b_{1}=\cdots=b_{k_{1}} \neq b_{k_{1}+1}=\cdots=b_{k_{1}+k_{2}} \neq \cdots \neq b_{k_{1}+\cdots+k_{r-1}+1}=\cdots=b_{n}
$$

holds on the whole of $M$.

Note that any sub-Riemannian or sub-Lorentzian structure fulfils the regularity condition at least on an open subset of $M$. Clearly, the functions $b_{i}$ are related to either real or purely imaginary eigenvalues 
of the operator $J$. In fact, if $(M, H, g)$ is regular then $\tilde{J}$ has necessarily form (15). Let

$$
H^{i}=\operatorname{span}\left\{X_{j}, X_{n+j} \mid k_{1}+\ldots+k_{i-1}+1 \leq j \leq k_{1}+\ldots+k_{i}\right\} .
$$

Then all $H^{i}, i=1, \ldots, r$, are invariant with respect to $J$ and $H$ splits into the Whitney sum

$$
H=H^{1} \oplus \cdots \oplus H^{r} .
$$

Moreover, the groups $\mathcal{G}_{g, \omega}(q), q \in M$, split into the direct product

$$
\mathcal{G}_{g, \omega}(q) \simeq\left(S p\left(\left.\omega\right|_{H_{q}^{1}}\right) \cap O\left(\left.g\right|_{H_{q}^{1}}\right)\right) \oplus \cdots \oplus\left(S p\left(\left.\omega\right|_{H_{q}^{r}}\right) \cap O\left(\left.g\right|_{H_{q}^{r}}\right)\right) .
$$

All groups $\mathcal{G}_{g, \omega}(q)$ are isomorphic under the regularity condition and will be shortly denoted $\mathcal{G}_{g, \omega}$. Consequently, the bundle $O_{H, g, \omega}(M)$ admits a reduction to a $\mathcal{G}_{g, \omega}$-structure which can be realized as the set of all such frames $\left(q ; v_{1}, \ldots, v_{2 n}, v_{0}\right) \in O_{H, g, \omega}$ that

$$
v_{j}, v_{n+j} \in H_{q}^{i}, \quad k_{1}+\ldots+k_{i-1}+1 \leq j \leq k_{1}+\ldots+k_{i},
$$

for $i=1, \ldots, r$. The presented considerations lead to the following

Theorem 4.2. Let $(M, H, g)$ be an oriented contact sub-pseudo-Riemannian manifold satisfying the regularity condition. Then

$$
\operatorname{dim} \mathfrak{I}(M, H, g) \leq 2 n+1+s_{1}^{2}+\left(k_{1}-s_{1}\right)^{2}+\cdots+s_{r}^{2}+\left(k_{r}-s_{r}\right)^{2},
$$

where $s_{i}=\frac{1}{2} \operatorname{rk}\left(H^{i} \cap \hat{H}\right)$.

Proof. Indeed, $\operatorname{dim} \mathfrak{I}(M, H, g) \leq \operatorname{dim} M+\operatorname{dim} \mathcal{G}_{g, \omega}$ and the result follows from Lemma 3.4 applied to each factor of $\mathcal{G}_{g, \omega}$ separately.

4.2. Left invariant regular structures. Now we are going to show that the upper bound on the dimension of the isometry group given in Theorem 4.2 is attained. To this end, fix positive real numbers $b_{i}$, $i=1, \ldots, n$, as in (24) and define the following multiplication on $\mathbb{R}^{2 n+1}$

$$
\begin{aligned}
& \left(x_{1}, \ldots, x_{n}, y_{1}, \ldots, y_{n}, z\right) *\left(x_{1}^{\prime}, \ldots, x_{n}^{\prime}, y_{1}^{\prime}, \ldots, y_{n}^{\prime}, z^{\prime}\right)= \\
& \left(x_{1}+x_{1}^{\prime}, \ldots, x_{n}+x_{n}^{\prime}, y_{1}+y_{1}^{\prime}, \ldots, y_{n}+y_{n}^{\prime}, \frac{1}{2} \sum_{i=1}^{n} b_{i}\left(y_{i} x_{i}^{\prime}-y_{i}^{\prime} x_{i}\right)\right) .
\end{aligned}
$$

The multiplication (26) can be treated as a deformation of the standard multiplication (20). Now it is not difficult to see that the left invariant vector fields with respect to this multiplication are given by formulae

$$
X_{i}=\frac{\partial}{\partial x_{i}}+\frac{b_{i}}{2} y_{i} \frac{\partial}{\partial z}, \quad Y_{i}=\frac{\partial}{\partial y_{i}}-\frac{b_{i}}{2} x_{i} \frac{\partial}{\partial z} .
$$


Let $H=\operatorname{span}\left\{X_{1}, Y_{1}, \ldots, X_{n}, Y_{n}\right\}$ and define metric $g$ by declaring the basis $X_{1}, Y_{1}, \ldots, X_{n}, Y_{n}$ to be orthonormal with

$$
g\left(X_{i}, X_{i}\right)=p_{i}, \quad g\left(Y_{i}, Y_{i}\right)=r_{i}
$$

where $p_{i}, r_{i} \in\{-1,+1\}$ depending on the index of the metric, $i=$ $1, \ldots, n$. It clear that the canonical contact form is

$$
\alpha=d z-\sum_{i=1}^{n}(1 / 2) b_{i}\left(y_{i} d x_{i}-x_{i} d y_{i}\right)
$$

and

$$
\omega=\sum_{i=1}^{n} b_{i} d x_{i} \wedge d y_{i} .
$$

It follows from the construction that the left translations with respect to (26) are isometries of $\left(\mathbb{R}^{2 n+1}, H, g\right)$, because vector fields (27) are left invariant. Moreover, any $\sigma \in \mathcal{G}_{g, \omega}$ decomposes according to the splitting (25). Performing similar calculations as in Lemma 3.5 for each factor of this decomposition one can prove

Proposition 4.3. The group of orientation preserving isometries of the left invariant contact sub-pseudo-Riemannian structure $(H, g)$ constructed above on $\mathbb{R}^{2 n+1}$ is isomorphic to

$$
\mathbb{R}^{2 n+1} \ltimes \mathcal{G}_{g, \omega},
$$

where $\mathcal{G}_{g, \omega}$ is given by (25).

\section{General Case}

5.1. Symbol algebra. Let $(M, H, g)$ be an oriented contact sub-pseudoRiemannian manifold. Let $\mathfrak{g}(H)(q)$ be the symbol algebra of $H$ at point $q \in M$. It is a two-step nilpotent graded Lie algebra

$$
\mathfrak{g}(H)(q)=\mathfrak{g}_{-1}(q) \oplus \mathfrak{g}_{-2}(q)
$$

where

$$
\mathfrak{g}_{-1}(q)=H_{q}, \quad \mathfrak{g}_{-2}(q)=T_{q} M / H_{q}
$$

The Lie bracket $\mathfrak{g}_{-1}(q) \wedge \mathfrak{g}_{-1}(q) \rightarrow \mathfrak{g}_{-2}(q)$ is defined in terms of the Lie bracket of vector fields on $M$ as follows. Let $v, w \in \mathfrak{g}_{-1}(q)$ and let $X_{v}$ and $X_{w}$ be two extensions of $v$ and $w$, respectively, to sections of $H$ in a neighbourhood of $q$. Then

$$
[v, w]=\left[X_{v}, X_{w}\right](q) \bmod H_{q}
$$

does not depend on the extension and defines the Lie bracket in $\mathfrak{g}(H)(q)$. Clearly, the Lie algebra $\mathfrak{g}(H)(q)$ does not depend on $q$. Moreover the 
dual space $\mathfrak{g}_{-2}(q)^{*}$ can be identified with $H_{q}^{\perp} \subset T_{q}^{*} M$ spanned by the contact form $\alpha_{q}$. It follows that

$$
\alpha_{q}([v, w])=\omega_{q}(v, w)
$$

i.e. the Lie algebra structure is determined by the symplectic form $\omega$.

The symbol algebra $\mathfrak{g}(H, g)(q)$ of $H$ equipped with $g$ at point $q \in M$ is defined as follows

$$
\mathfrak{g}(H, g)(q)=\mathfrak{g}(H)(q) \oplus \mathfrak{g}_{0}(q)
$$

where $\mathfrak{g}_{0}(q)$ is the algebra of matrices $A \in \mathfrak{g l}(\mathfrak{g}(H)(q))$ preserving the metric $g$, i.e.

$$
g(A v, w)+g(v, A w)=0
$$

and the Lie bracket on $\mathfrak{g}_{-1}(q)$, i.e.

$$
[A v, w]+[v, A w]=A[v, w] .
$$

Since the Lie bracket is encoded in terms of $\omega$ it follows that $\mathfrak{g}_{0}(q)$ is the Lie algebra of the Lie group $\mathcal{G}_{g, \omega}(q)$ and actually can be thought of as a sub-algebra of $\mathfrak{g l}\left(\mathfrak{g}_{-1}(q)\right)$. Defining

$$
[A, v]=A v
$$

for $v \in \mathfrak{g}_{-1}(q)$ we get that $\mathfrak{g}(H, g)(q)$ is a graded Lie algebra. We refer to [15] for more information on the symbol algebras of distributions and related structures.

5.2. Prolongation. The first prolongation of $\mathfrak{g}(H, g)(q)$ is defined as

$$
\operatorname{pr}_{1}(\mathfrak{g}(H, g)(q))=\mathfrak{g}(H, g)(q) \oplus \mathfrak{g}_{1}(q),
$$

where $\mathfrak{g}_{1}(q)$ is the set of all Lie algebra derivations $\mathfrak{g}(H) \rightarrow \mathfrak{g}(H, g)$ increasing the gradation by 1 , i.e. any $A \in \mathfrak{g}_{1}(q) \operatorname{maps} \mathfrak{g}_{-1}(q)$ to $\mathfrak{g}_{0}(q)$ and $\mathfrak{g}_{-2}(q)$ to $\mathfrak{g}_{-1}(q)$ such that

$$
A([v, w])=A(v) w-A(w) v
$$

for all $v, w \in \mathfrak{g}_{-1}(q)$. Note that $\operatorname{dim} \mathfrak{g}_{-2}=1$ thus for any $A \in \mathfrak{g}_{1}(q)$ the image $A\left(\mathfrak{g}_{-2}\right)$ is a one- or zero-dimensional subspace of $\mathfrak{g}_{-2}$.

Higher prolongations of $\mathfrak{g}(H, g)(g)$ are defined by induction, similarly to the first prolongation, as Lie algebra derivations increasing the gradation by $k \in \mathbb{N}$. We get

$$
\operatorname{pr} \mathfrak{g}(H, g)(q)=\mathfrak{g}(H, g)(q) \oplus \bigoplus_{k \in \mathbb{N}} \mathfrak{g}_{k}(q)
$$

and one equips pr $\mathfrak{g}(H, g)(q)$ with the structure of a graded Lie algebra in a natural way. However we shall not describe the structure in detail because we have the following 
Lemma 5.1. The first prolongation of $\mathfrak{g}(H, g)(q)$ is trivial. Consequently

$$
\operatorname{pr} \mathfrak{g}(H, g)(q)=\mathfrak{g}(H, g)(q)
$$

Proof. Let $\alpha_{q}^{*} \in \mathfrak{g}_{-2}(q)$ be a vector dual to the contact form $\alpha_{q}$, i.e. $\alpha_{q}\left(\alpha_{q}^{*}\right)=1$. Choose $A \in \mathfrak{g}_{1}(q)$ and denote $v_{A}=A\left(\alpha_{q}^{*}\right)$. Let $\left(v_{1}, \ldots, v_{2 n}\right)$ be an orthonormal basis of $H_{q}$ that puts $J$ into the canonical Kronecker form (14). Then (28) reads

$$
A\left(v_{i}\right) v_{j}-A\left(v_{j}\right) v_{i}=g\left(J\left(v_{i}\right), v_{j}\right) v_{A} .
$$

Since $\left(v_{1}, \ldots, v_{2 n}\right)$ is orthonormal it follows that all $A\left(v_{i}\right), i=1, \ldots, 2 n$, are orthonormal matrices in $\mathfrak{s o}(l, 2 n-l)$. Now, for a fixed value of $v_{A}$ there is unique $A$ that solves (29) in $\mathfrak{s o}(l, 2 n-l)$, where $l=\operatorname{ind}(g)$. This follows from the uniqueness of the Levi-Civita connection of a pseudoRiemannian metric which is equivalent to the algebraic fact that the system

$$
A\left(v_{i}\right) v_{j}-A\left(v_{j}\right) v_{i}=0
$$

has unique solution $A=0$ in the algebra $\mathfrak{s o}(l, 2 n-l)$. The unique solution to (29) is of the form

$$
A=\frac{1}{2} \sum_{i=1}^{2 k}\left(v_{A} \cdot J\left(v_{i}\right)^{T}\right) v_{i}^{*}
$$

where $\left(v_{1}^{*}, \ldots, v_{2 n}^{*}\right)$ are dual to $\left(v_{1}, \ldots, v_{2 n}\right)$ with respect to $g$ and $v_{A}$. $J\left(v_{i}\right)^{T}=A\left(v_{i}\right)$ is a rank-one square matrix $A\left(v_{i}\right)=\left(a_{j k}^{i}\right)_{j, k=1, \ldots, 2 n}$ with entries $a_{j k}^{i}=v_{j}^{*}\left(v_{A}\right) v_{k}^{*}\left(J\left(v_{i}\right)\right)$. Now, since all $A\left(v_{i}\right)$ are orthonormal it follows that $v_{j}^{*}\left(v_{A}\right) v_{j}^{*}\left(J\left(v_{i}\right)\right)=0$ for any $j=1, \ldots, 2 n$. But, for any $i$ there is $j$ such that $v_{j}^{*}\left(J\left(v_{i}\right)\right) \neq 0$. Thus we get that $v_{j}^{*}\left(v_{A}\right)=0$, for $j=1, \ldots, 2 n$. Consequently, $v_{A}=0$. This reduces (29) to (30). Hence $A=0$, because this is the unique solution to (30) as was explained above.

Now we are able to apply Theorem 1 of [13] and get

Theorem 5.2. Let $(M, H, g)$ be an oriented contact sub-pseudo-Riemannian manifold. Then the dimension of the algebra of the infinitesimal symmetries of $(M, H, g)$ is estimated from above by

$$
\operatorname{dim} M+\inf _{q \in M} \operatorname{dim} \mathcal{G}_{g, \omega}(q) .
$$

Proof. We have pr $\mathfrak{g}(H, g)(q)=\mathfrak{g}(H, g)(q)$. Thus $\operatorname{dim} \operatorname{pr} \mathfrak{g}(H, g)(q)=$ $\operatorname{dim} M+\operatorname{dim} \mathcal{G}_{g, \omega}(q)$ since $\mathfrak{g}_{0}(q)$ is the Lie algebra of $\mathcal{G}_{g, \omega}(q)$. All the prolongations are finite. Therefore, by [13], we have that the dimenison 
of the algebra of infinitesimal symmetries is estimated from above by $\inf _{q \in M} \operatorname{dim} \operatorname{pr} \mathfrak{g}(H, g)(q)$.

5.3. Proof of Theorem 1.2. If $(M, H, g)$ is an oriented sub-pseudoRiemannian manifold then it suffices to consider isometries preserving the orientation because $\operatorname{dim} \mathfrak{I}(M, H, g)=\operatorname{dim} \mathfrak{I}_{0}(M, H, g)$. The dimension of $\mathfrak{I}_{0}(M, H, g)$ equals to the dimension of the algebra of infinitesimal isometries. Therefore we can apply Theorem 5.2. The maximal possible dimension of $\mathcal{G}_{g, \omega}$ is computed in Lemma 3.4.

If $(M, H, g)$ is not oriented then we consider a double cover $\tilde{M}$ of $M$ consisting of pairs $\left(q, \alpha_{q}\right)$ where $q \in M$ and $\alpha_{q}$ is one of the two normalised co-vectors in $T_{q}^{*} M$ annihilating $H(q)$. Then $\tilde{M}$ carries a canonical lift $(\tilde{H}, \tilde{g})$ of the structure $(H, g)$ and the structure $(\tilde{M}, \tilde{H}, \tilde{g})$ is oriented, because $\left(q, \alpha_{q}\right) \mapsto \alpha_{q}$ defines a global contact form on $\tilde{M}$ annihilating $\tilde{H}$. Moreover, any isometry of the original structure $(M, H, g)$ defines an isometry of $(\tilde{M}, \tilde{H}, \tilde{g})$ and thus $\operatorname{dim} \mathfrak{I}(M, H, g) \leq$ $\operatorname{dim} \mathfrak{I}(\tilde{M}, \tilde{H}, \tilde{g})$. Therefore, the estimate in the not oriented case follows from the estimate in the oriented case.

Acknowledgements. The work of Wojciech Kryński has been partially supported by the Polish National Science Centre grant DEC2011/03/D/ST1/03902.

\section{REFERENCES}

[1] A. Agrachev, Exponential mappings for contact sub-Riemannian structures, J. Dynam. Control Systems 2, No. 3 (1996), 321-356.

[2] A. Agrachev, El-H. Chakir El-A., J.P. Gauthier, Sub-Riemannian metrics on $R^{3}$, Canadian Mathematical Society Conference Proceedings, Vol. 25, 1998.

[3] A. Agrachev, D. Barilari, Sub-Riemannian structures on 3D Lie groups, J. Dynam. Control Systems 18, (2012).

[4] M. Gromov, Carnot-Carathéodory spaces seen from within, Sub-Riemannian geometry, Progr. Math., Vol. 144, Birkhäuser, Basel, 1996, 79-323.

[5] M. Grochowski, The structure of reachable sets for affine control systems induced by generalized Martinet sub-Lorentzian metrics, ESAIM Control Optim. Calc. Var. 18 (2012), 1150-1177.

[6] M. Grochowski, Remarks on global sub-Lorentzian geometry, Anal. Math. Phys. 3, No. 4 (2013), 295-309.

[7] M. Grochowski, W. Kryński, Invariants of contact sub-pseudo-Riemannian structures and Einstein-Weyl geometry, submitted (2015).

[8] M. Grochowski, B. Warhurst, Invariants and Infinitesimal Transformations for Contact Sub-Lorentzian Structures on 3-Dimensional Manifolds, SIGMA 11 (2015), 031.

[9] E. Grong, A. Vasilev, Sub-Riemannian and sub-Lorentzian geometry on SU $(1,1)$ and on its universal cover, J. Geom. Mech. 3, No. 2 (2011), 225-260. 
[10] S. Kobayashi, Transformation groups in differential geometry, Springer-Verlag, New York-Heidelberg, 1972.

[11] A. Korolko, I. Markina, Geodesics on H-type quaternion groups with subLorentzian metric and their physical interpretation, Complex Anal. Oper. Theory 4 , No. 3 (2010), 589-618.

[12] B. Kruglikov, Finite-dimensionality in Tanaka theory, Ann. Inst. H. Poincaré Anal. Non Linéaire 28, No. 1 (2011), 75-90.

[13] B. Kruglikov, Symmetries of filtered structures via filtered Lie equations, J. Geom. Phys., Vol. 85 (2014), 164-170.

[14] R. Montgomery, A Tour of Subriemannian Geometries, Their Geodesics and Applications, AMS, 2006.

[15] N. Tanaka, On differential systems, graded Lie algebras and pseudogroups, J. Math. Kyoto Univ., Vol. 10 (1970), 1-82

[16] R. Thompson, Pencils of Complex and Real Symmetric and Skew Matrices, Linear Algebra and Its Applications, Vol. 147 (1991), 323-371.

Faculty of Mathematics and Natural Sciences, Cardinal Wyszyński University, 00-956 WARszaWA, POlAnd

E-mail address: m.grochowski@uksw.edu.pl

Institute of Mathematics, Polish Academy of Sciences, Ul. Śniadeckich 8, 00-956 WARSZAWA, POLAND

E-mail address: w.krynski@impan.pl 